\title{
SIERPINSKI SETS AND STRONG FIRST CATEGORY
}

\author{
JAKUB JASINSKI AND TOMASZ WEISS
}

(Communicated by Andreas R. Blass)

\begin{abstract}
We prove that if $S$ is a Sierpinski set and $N \subseteq \mathbb{R}$ is an $F_{\sigma}$ set of measure zero, then $(N+t) \cap S=\varnothing$ for some $t \in \mathbb{R}$. A similar result holds for generalized Sierpinski sets under Martin's Axiom.
\end{abstract}

\section{INTRODUCTION}

An uncountable set $S \subseteq \mathbb{R}$ is called a Sierpinski set if $|N \cap S| \leq \omega$ whenever $N \subseteq \mathbb{R}$ is of measure zero. We say that $X \subseteq \mathbb{R}$ has strong first category (SFC), if, for any measure-zero set $N \subseteq \mathbb{R}$, there exists $t \in \mathbb{R}$ such that $(N+t) \cap X=\varnothing$. It is well known that Lusin sets, the category analog of the Sierpinski sets, have strong measure zero (see [M] for details). This leads to the question posed by F. Galvin [M, p. 210]: Do all Sierpinski sets have SFC? It is known that under $\mathrm{CH}$ there are Sierpinski sets which have SFC. Simply let $\left\{N_{\alpha}\right\}_{\alpha<\omega}$ be all $G_{\delta}$ measure zero sets and inductively pick

$$
s_{\alpha} \in \mathbb{R} \backslash \bigcup_{\beta<\alpha}\left(\left(F_{\beta} \cup\left\{s_{\beta}\right\}\right) \cup\left(F_{\beta}+t_{\beta}\right)\right),
$$

where $t_{\alpha}$ is such that $\left\{s_{\beta}: \beta \subseteq \alpha\right\} \cap\left(F_{\alpha}+t_{\alpha}\right)=\varnothing$. Then $S=\left\{s_{\alpha}: \alpha<\omega_{1}\right\}$ is an SFC Sierpinski set. Recently Bartoszynski and Ihoda proved that under $\mathrm{CH}$ every Sierpinski set is a union of two SFC sets (although it is not known if the union of two SFC sets always has SFC) and that $\operatorname{Con}(\mathrm{ZF}) \Rightarrow$ Con $(\mathrm{ZFC}+$ there are Sierpinski sets + all Sierpinski sets have SFC) [BI].

\section{MAIN RESUlt}

Let $\mu$ be Lebesgue measure on $\mathbb{R}$ and let $L_{0}=\{N \subseteq \mathbb{R}: \mu(N)=0\}$. For $X, Y \subseteq \mathbb{R} X \pm Y=\{x \pm y: x \in X$ and $y \in Y\}$.

The following is a partial answer to Galvin's question.

Theorem 1. If $N \in L_{0}$ is an $F_{\sigma}$ set and $S$ a Sierpinski set, then there exists $t \in \mathbb{R}$ such that $(N+t) \cap S=\varnothing$.

Received by the editors October 20, 1989 and, in revised form, February 12, 1990.

1980 Mathematics Subject Classification (1985 Revision). Primary 04A15; Secondary 28 A 15. 
The proof is based on the following simple observation:

Lemma 1. Let $N \in L_{0}$ and $Q \subseteq \mathbb{R}$ be such that

(1) $N+Q \in L_{0}$ and

(2) $N$ can be shifted out of every countable set by a member of $Q$; i.e., for every countable set $X \subseteq \mathbb{R}, Q \nsubseteq X-N$.

Then $N$ can be shifted out of every Sierpinski set.

Proof. Let $S$ be a Sierpinski set and let $X=S \cap(N+Q)$, so that $X$ is countable. Then any member of $Q$ that shifts $N$ out of $X$ shifts $N$ out of $S$; i.e., if $t \in Q$ and $(N+t) \cap X=\varnothing$, then $(N+t) \cap S=\varnothing$.

[EKM, Lemma 9] shows that, if $N \in L_{0}$, then there exists a perfect set $Q$ such that $N+Q \in L_{0}$. It follows that every Sierpinski set can be shifted out of any measure-zero set $N$ such that countably many translates of $N$ cannot cover any perfect set. Recall that a set $X \subseteq \mathbb{R}$ has universal measure zero iff, for all atomless measures $\nu$ on the Borel sets, there is a Borel set of $\nu$-measure zero covering $X$. Also recall that $X$ has property $s_{0}$ iff for any perfect set $P$ there exists a perfect set $Q \subseteq P$ disjoint from $X$. It is well known that neither universal-measure-zero sets nor sets with property $s_{0}$ can cover a perfect set by countably many translates (for this and other properties of these sets see [M]). We have the following:

Corollary. If $M \subseteq \mathbb{R}$ is universally null or $M \in L_{0}$ and has property $s_{0}$ and $S$ is a Sierpinski set, then $(M+t) \cap S=\varnothing$ for some $t \in \mathbb{R}$.

For our main result, we need $\left\{p_{k}\right\}_{k \in \omega}$-expansions. Let $\left\{p_{k}\right\}_{k \in \omega}$ be a sequence of natural numbers each greater than 1 . Every real number $x \in[0,1)$ has a unique $\left\{p_{k}\right\}_{k \in \omega}$-expansion of the form $x=\sum_{k \in \omega} \frac{x_{k}}{p_{0} \cdot p_{1} \cdots \cdot p_{k}}$, where $x_{k} \in$ $p_{k}$ and $x_{k} \neq p_{k}-1$ for infinitely many $k$. (We identify $p_{k}$ with $\{0,1,2, \ldots$, $\left.p_{k}-1\right\}$.) In what follows, $x_{k}$ will be denoted by $(x)_{k}$.

Lemma 2. Let $N \in L_{0}$ be an $F_{\sigma}$ subset of $[0,1)$. Then there exists a sequence $\left\{p_{k}\right\}_{k \in \omega}$ and a sequence $\left\{\Delta_{k}\right\}_{k \in \omega}$ such that, for all $k \in \omega$,

$$
p_{k} \geq 6, \Delta_{k} \subseteq p_{k} \text { and }\left|\Delta_{k}\right| \leq \frac{p_{k}}{3},
$$

and there exists a countable set $Y \subseteq \mathbb{R}$ such that $N \subseteq D+Y$, where

$$
D=\left\{\sum_{k \in \omega} \frac{d_{k}}{p_{0} \cdot p_{1} \cdots \cdot p_{k}}: d_{k} \in \Delta_{k}\right\} \text {. }
$$

Proof. Let $N_{0}, N_{1}, \ldots \subseteq[0,1)$ be closed sets such that $N=\bigcup_{k \in \omega} N_{k}$. Define $I_{n}^{i}=\left[\frac{i}{2^{n}} \frac{i+1}{2^{n}}\right)$, where $n \in \omega$ and $i \in 2^{n}$. Also, let $Q_{k}(n)=\left|\left\{i: I_{n}^{i} \cap N_{k} \neq \varnothing\right\}\right|$. It is not hard to show that, since $N_{k}$ is closed (in $\mathbb{R}$ ), $\lim _{n \rightarrow \infty} \frac{Q_{k}(n)}{2^{n}}=0$. Using this fact, we define $\left\{p_{k}\right\}_{k \in \omega}$ by induction.

Let $m \in \omega$ be such that $2^{m} \geq 6$ and $\frac{Q_{0}(m)}{2^{m}} \leq \frac{1}{3}$. Put $p_{0}=2^{m}$ and 


$$
\begin{gathered}
\Delta_{0}=\left\{i: I_{m}^{i} \cap N_{0} \neq \varnothing\right\} . \text { At stage } k+1, \text { consider } I_{k}=\left[0, \frac{1}{p_{0} \cdot p_{1} \cdot \cdots \cdot p_{k}}\right) \text { and } \\
B_{k}=\left(N_{k} \cup N_{k}-\frac{1}{p_{0} \cdot p_{1} \cdot \ldots \cdot p_{k}} \cup \ldots \cup N_{k}-\frac{p_{0} \cdot p_{1} \cdot \ldots \cdot p_{k}-1}{p_{0} \cdot p_{1} \cdot \ldots \cdot p_{k}}\right) \cap I_{k} .
\end{gathered}
$$

Now pick $m$ so that $2^{m} \geq 6$. Then, if we partition $I_{k}$ into $2^{m}$ equal subintervals, at most $\frac{2 m}{3}$ of them have nonempty intersections with $B_{k}$. Let $p_{k+1}=2^{m}$ and $\Delta_{k+1} \subseteq p_{k+1}$ be a set of indices of those subintervals whose intersections with $B_{k}$ are nonempty. Clearly conditions (1) are satisfied.

Now suppose $D$ is as in Lemma 2 and $a \in N_{k_{0}}$ for some $k_{0} \in \omega$. For $k \geq k_{0},(a)_{k} \in \Delta_{k}$. Therefore, if we let $Y$ be the set of all real numbers from $(-1,1)$ with finite $\left\{p_{k}\right\}_{k \in \omega}$-expansions, then $a \in D+Y$.

We now have to modify [EKM, Lemma 9].

Lemma 3. Let $\left\{p_{k}\right\}_{k \in \omega}$ be a bounded sequence of natural numbers greater than 1. For each set $K \subseteq \omega$, let

$$
Q_{K}=\left\{\sum_{k \in \omega} \frac{q_{k}}{p_{0} \cdot p_{1} \cdots p_{k}}: q_{k} \in p_{k} \text { if } k \in K \text { and } q_{k}=0 \text { otherwise }\right\} .
$$

Given $N \in L_{0}$, there exists an infinite set $K \subseteq \omega$ such that $N+Q_{K} \in L_{0}$.

Proof. The proof is almost the same as the original proof of Erdös, Kunen, and Mauldin. Note that if $f: \omega \rightarrow \omega$ is an increasing function, $I \subseteq \mathbb{R}$ is an interval, and $n \in \omega$, then $\mu\left(I+Q_{\operatorname{ran}(f)}\right) \leq p_{f(0)} \cdot p_{f(1)} \cdots \cdot p_{f(n)}\left(\mu(I)+\frac{1}{p_{0} \cdot p_{1} \cdots \cdot p_{f(n)}}\right)$. By making $f$ increase rapidly enough we make $p_{f(0)} \cdot p_{f(1)} \cdots \cdot p_{f(n)} / p_{0} \cdot p_{1} \cdots \cdots$ $p_{f(n)}$ as small as desired so that all steps in the proof given in [EKM] may be performed here without major changes. We leave the details to the reader. However, in the case relevant to the proof of Theorem $1, p_{k}=2^{r_{k}}$ for some $r_{k} \in \omega$. For such $p_{k}$, Lemma 3 follows easily. By [EKM, Lemma 9], we have an infinite set $H \subseteq \omega$ such that if

$$
B_{H}=\left\{\sum_{k \in \omega} \frac{q_{k}}{2^{k}} q_{k} \in\{0,1\} \text { if } k \in H \text { and } q_{k}=0 \text { otherwise }\right\},
$$

then $N+B_{H} \in L_{0}$ and, if $b \in \omega$ is a bound for $\left\{r_{k}\right\}_{k \in \omega}$ and $H_{k}=\{k+i: i<$ $b\}$, then there exists an infinite set $X \subseteq \omega$ such that, whenever $k, 1 \in X$, then $H_{k} \cap H_{1}=\varnothing$ and $H_{k} \subseteq H$. Now simply let $K=\{k+b: k \in X\}$, and observe that $Q_{k} \subseteq B_{H}$.

Proof of Theorem 1. Let $N \in L_{0}$ be an $F_{\sigma}$ set. Then $N^{\prime}=\left(\bigcup_{n \in \omega}(N \pm n)\right) \cap$ $[0,1)$ is also an $F_{\sigma}$ set, so that by Lemma 2 there exist sequences $\left\{p_{k}\right\}_{k \in \omega}$, $\left\{\Delta_{k}\right\}_{k \in \omega}$ and a set $D$ such that $N^{\prime} \subseteq D+Y$ for some countable $Y \subseteq \mathbb{R}$. Since in the proof of Lemma 2 the sets $N_{k}$ can be made as small as desired, we may assume that the sequence $\left\{p_{k}\right\}_{k \in \omega}$ is bounded. Therefore, by Lemma 3, we have an infinite set $K=\left\{k_{0}, k_{1}, k_{2}, \ldots\right\} \subseteq \omega$, so that $Q_{K}+N \in L_{0}$. By Lemma 1, it suffices to show that $N$ does not cover $Q_{K}$ by countable translations. Since $N^{\prime}$ as well as $N$ may be covered by countably many translations of $D$, let us now 
show that for any countable $X \subseteq \mathbb{R}, X+D \nsupseteq Q_{K}$. Let $X=\left\{x^{(n)}: n \in \omega\right\}$. By assuming that the addition in $X+D$ is modulo 1 , we may consider $X \subseteq[0,1)$. For each $i \in \omega$, pick $q_{k_{i}} \in\left(p_{k_{i}}-1\right) \backslash\left[\left(\Delta_{k_{i}} \cup \Delta_{k_{i}} \oplus 1\right) \oplus\left(x^{(i)}\right)_{k_{i}}\right]$, where $\oplus$ signifies algebraic addition modulo $p_{k_{i}}$. Set $q_{k}=0$ for $k \notin K$ so that the number $q=\sum_{k \in \omega} \frac{q_{k}}{p_{0} \cdot p_{1} \cdots \cdot p_{k}}$ is in $Q_{K}$ and is different from all numbers in $X+D$.

A similar result holds for generalized Sierpinski sets under Martin's Axiom (MA). Recall that $S \subseteq \mathbb{R}$ is a generalized Sierpinski set if $|S|=c$ and $|S \cap N|<$ $c$ whenever $N \in L_{0}$. Clearly, Lemma 1 remains true for generalized Sierpinski sets if "countable $X$ " is replaced by " $|X|<c$."

Theorem 2. Assume MA. If $N \in L_{0}$ is an $F_{\sigma}$ set, then for every generalized Sierpinski set $S$ there exists a $t \in \mathbb{R}$ such that $(N+t) \cap S=\varnothing$.

Proof. Let $N \in L_{0}$ be an $F_{\sigma}$ set. It suffices to show that $N$ satisfies the generalized Lemma 1 as shown above. Let $N^{\prime},\left\{p_{k}\right\}_{k \in \omega},\left\{\Delta_{k}\right\}_{k \in \omega}$, and $K$ be as in the proof of Theorem 1. We would like to show that $D+X \nsupseteq Q_{K}$ for any $X \subseteq \mathbb{R}$, where $|X|<c$. Let $\mathbf{P}=\{(f, E): E \subseteq X$ is a finite subset and $f$ is a finite function, $\operatorname{dom}(f) \subseteq \omega, f(i) \in p_{1}$ and for every $e \in E$ there exists an $i \in K \cap \operatorname{dom}(f)$ such that $f(i) \in p_{1} \backslash(D+e)_{1}$ and $f(i)=0$ for $i \in \operatorname{dom}(f) \backslash K\}$. Define a partial order on $\mathbf{P}$ simply as $\left(f_{2}, E_{2}\right) \leq\left(f_{1}, E_{1}\right)$ if $f_{2} \supseteq f_{1}$ and $E_{2} \supseteq E_{1}$. Clearly $(\mathbf{P}, \leq)$ is a ccc poset and $D_{F}=\{(f, E): E \supseteq F\}$ and $D_{n}=\{(f, E): n \in \operatorname{dom}(f)\}$ are dense subsets of $\mathbf{P}$ for all finite $F \subseteq X$ and $n \in \omega$. By Martin's Axiom, there exists a filter $G \subseteq \mathbf{P}$ which meets all $D_{F}$ and $D_{n}$. Let $q=\bigcup\{f:(f, E) \in G\}$. It is easy to see that

$$
\sum_{k \in \omega} \frac{q(k)}{p_{0} \cdot p_{1} \cdots \cdot p_{k}} \in Q_{k} \backslash(D+X) .
$$

Added in proof. I. Reclaw and A. W. Miller have obtained independently a short, elegant proof of the following theorem.

Theorem. If $S$ is a Sierpinski set and $F$ is a $F_{\sigma}$ set of measure zero, then $\{x:(x+F) \cap S=\varnothing\}$ is comeager.

T. Bartoszynski and I. Reclaw pointed out that for some dense $G_{\delta}$ sets $N$ there are sets $Q$ such that conditions (1) and (2) of Lemma 1 hold.

\section{REFERENCES}

[BI] T. Bartoszynski and J. I. Ihoda, On Sierpinski sets, Proc. Amer. Math. Soc. 108 (1990), 507-512.

[EKM] P. Erdös, K. Kunen, and R. D. Mauldin, Some additive properties of sets of real numbers, Fund. Math. 113 (1981), 187-194.

[M] A. W. Miller, Special subsets of the real line, in Handbook of set-theoretic topology (K. Kunen and J. E. Vaughan, eds.), Elsevier Science Publishers B. V., 1984.

Department of Mathematics, University of Scranton, Scranton, Pennsylvania 18510

Department of Mathematics, Pennsylvania State University, University Park, PennSYLVANIA 16802 